\title{
Oscillation of second order difference equation with several super-linear neutral terms
}

\section{S. Mehar Banu ${ }^{1}$ and S. Nalini ${ }^{2 *}$}

\section{"Correspondence:}

nalininsit@gmail.com

${ }^{2}$ Research and Development Center, Bharathiar University, Coimbatore, India

Full list of author information is

available at the end of the article

\section{Springer}

\begin{abstract}
In this article,we present some new sufficient conditions for the oscillation of all solutions of a second order difference equation with several super-linear neutral terms. The results obtained here extend or complement some of the known results reported in the literature. Examples illustrating the importance of the main results are included.
\end{abstract}

MSC: $39 \mathrm{~A} 11$

Keywords: Second order; Difference equation; Super-linear neutral terms; Oscillation

\section{Introduction}

This paper deals with the oscillatory behavior of solutions of second order difference equation with several super-linear neutral terms of the form

$$
\Delta(a(n) \Delta z(n))+q(n) x^{\beta}(n+1-\ell)=0, \quad n \geq n_{0} \geq 0,
$$

where $z(n)=x(n)+\sum_{i=1}^{m} p_{i}(n) x^{\alpha_{i}}\left(n-k_{i}\right)$, subject to the following conditions:

$\left(H_{1}\right) \alpha_{i} \geq 1$ for $i=1,2, \ldots, m$ and $\beta$ are ratios of odd positive integers;

$\left(H_{2}\right)\{a(n)\},\{q(n)\}$, and $\left\{p_{i}(n)\right\}$ for $i=1,2, \ldots, m$ are positive real sequences for $n \geq n_{0}$;

$\left(H_{3}\right) \quad \ell$ and $k_{i}(i=1,2, \ldots, m)$ are positive integers.

Let $\theta=\max \left\{\ell, k_{1}, k_{2}, \ldots, k_{m}\right\}$. By a solution of Eq. (1.1) we mean a real sequence $\{x(n)\}$ defined for $n \geq n_{0}-\theta$ and satisfying Eq. (1.1) for all $n \geq n_{0}$. We consider only those solutions $\{x(n)\}$ of Eq. (1.1) which satisfy $\sup \{|x(n)|: n \geq N\}>0$ for all $n \geq N$, and assume that Eq. (1.1) possesses such solutions. As usual, a solution of Eq. (1.1) is called oscillatory if it is neither eventually positive nor eventually negative; otherwise it is called nonoscillatory. If all the solutions are oscillatory, then the equation itself is called oscillatory.

In the past few years there has been an increasing interest in studying the oscillatory behavior of difference equations with linear neutral terms, see $[1,3,7,9,10,12,13,15$, 16] and the references contained therein. As indicated by Hale [4], MacDonald [8], and others, neutral equations having a nonlinearity in the neutral arise in many applications in population dynamics, in the study of vibrating masses attached to an elastic bar, electic networks containing lossless transmission lines as in high-speed computers, and in the solutions of variational problems with time delays.

(c) The Author(s) 2018. This article is distributed under the terms of the Creative Commons Attribution 4.0 International License (http://creativecommons.org/licenses/by/4.0/), which permits unrestricted use, distribution, and reproduction in any medium, provided you give appropriate credit to the original author(s) and the source, provide a link to the Creative Commons license, and indicate if changes were made. 
In view of the above discussion, in this paper we choose to examine the oscillatory behavior of such type of difference equations because similar properties for difference equations with linear neutral term received great attention of the researchers. The oscillatory behavior of second order difference equations with sub-linear neutral term, that is, $x^{\alpha}(n-k)$ with $0<\alpha<1$, was studied in $[2,6,14,17,18]$, and therefore in this paper we investigate the oscillatory properties of solutions of Eq. (1.1) with super-linear neutral terms, that is, $x^{\alpha_{i}}\left(n-k_{i}\right)$ with $\alpha_{i}>1$ for $i=1,2, \ldots, m$.

In [7], the author considered equation of the form

$$
\Delta\left(x_{n}-p_{n} x_{n-k}^{\alpha}\right)+q_{n} x_{n-\ell}^{\beta}=0, \quad n \geq n_{0}
$$

and studied its oscillatory behavior. In [5], the authors considered equation of the form

$$
\Delta\left(a_{n} \Delta\left(x_{n}+p_{n} x_{n-k}^{\alpha}\right)\right)+q_{n} x_{n-\ell}^{\beta}=0, \quad n \geq n_{0},
$$

where $\alpha>1$ and $\beta>0$ and discussed the oscillatory behavior of solutions.

Recently, in [9, 15], the authors considered Eq. (1.1) with $\alpha_{i}=1$ for all $i=1,2, \ldots, m$ and investigated the oscillatory behavior of all solutions in the two cases

$$
\sum_{n=n_{0}}^{\infty} \frac{1}{a(n)}=\infty
$$

and

$$
\sum_{n=n_{0}}^{\infty} \frac{1}{a(n)}<\infty .
$$

Motivated by the above observations, in this paper, we have obtained some new sufficient conditions for the oscillation of all solutions of Eq. (1.1) by using a Riccati type transformation, a summation averaging technique, and a comparison method. Thus the results obtained in this paper are new and extend those reported in [5, 9, 10, 15-18]. Examples are provided to illustrate the importance of the main results.

\section{Oscillation results}

In this section, we obtain sufficient conditions for the oscillation of all solutions of Eq. (1.1). Due to the form of our equation, we only need to give proofs for the case of eventually positive nonoscillatory solutions since the proofs for negative nonoscillatory solutions would be similar.

We begin with the following lemma.

Lemma 2.1 Let $\left(H_{1}\right)-\left(H_{3}\right)$ and condition (1.4) hold. If $\{x(n)\}$ is a positive solution of Eq. (1.1), then the corresponding function $\{z(n)\}$ satisfies

$$
z(n)>0, \quad \Delta z(n)>0, \quad \text { and } \quad \Delta(a(n) \Delta z(n))<0
$$

eventually. 
Proof The proof is similar to that of Lemma 1 of [18] and hence the details are omitted.

Lemma 2.2 Let $\{x(n)\}$ be an eventually positive solution of Eq. (1.1), and suppose (2.1) holds. Then there is an integer $N \geq n_{0}$ such that

$$
x(n) \geq(1-P(n)) z(n),
$$

where $P(n)=\sum_{i=1}^{m} M^{\alpha_{i}-1} p_{i}(n) R^{\alpha_{i}-1}(n)$ and $R(n)=\sum_{s=N}^{n-1} \frac{1}{a(s)}$ for all $n \geq N$ and for every constant $M>0$.

Proof From $\left(H_{2}\right)$ and the definition of $z(n)$, we have $z(n) \geq x(n)$ for all $n \geq n_{1} \geq n_{0}$. Further, from (2.1), we have

$$
z(n)=z\left(n_{1}\right)+\sum_{s=n_{1}}^{n-1} \frac{a(s) \Delta z(s)}{a(s)} \geq R(n) a(n) \Delta z(n),
$$

and hence $\Delta\left(\frac{z(n)}{R(n)}\right)<0$ for all $n \geq n_{1}$. That is, $\frac{z(n)}{R(n)}$ is decreasing for all $n \geq n_{1}$. Now

$$
\begin{aligned}
x(n) & =z(n)-\sum_{i=1}^{m} p_{i}(n) x^{\alpha_{i}}\left(n-k_{i}\right) \geq z(n)-\sum_{i=1}^{m} p_{i}(n) z^{\alpha_{i}}(n) \\
& \geq\left(1-\sum_{i=1}^{m} M^{\alpha_{i}-1} p_{i}(n) R^{\alpha_{i}-1}(n)\right) z(n),
\end{aligned}
$$

where we have used $z(n)$ is increasing and $\frac{z(n)}{R(n)} \leq M$ for all $n \geq N \geq n_{1}$.

Lemma 2.3 Let $\left(H_{1}\right)-\left(H_{3}\right)$ and condition (1.5) hold. If $\{x(n)\}$ is a positive solution of Eq. (1.1), then the corresponding sequence $\{z(n)\}$ satisfies one of the following two cases for all sufficiently large $n$ :

(1) $z(n)>0, \Delta z(n)>0, \Delta(a(n) \Delta z(n))<0$,

(2) $z(n)>0, \Delta z(n)<0, \Delta(a(n) \Delta z(n))<0$.

Proof The proof is similar to that of Lemma 2.1 of [15] and hence the details are omitted.

Lemma 2.4 Let $\{x(n)\}$ be a positive solution of Eq. (1.1) and the corresponding function $\{z(n)\}$ satisfies Case (2) of Lemma 2.3. Then there exists an integer $N \geq n_{0}$ such that

$$
x(n) \geq(1-Q(n)) z(n),
$$

where

$$
Q(n)=\sum_{i=1}^{m} K^{\alpha_{i}-1} p_{i}(n) \frac{A^{\alpha_{i}-1}\left(n-k_{i}\right)}{A(n)}
$$

and

$$
A(n)=\sum_{s=n}^{\infty} \frac{1}{a(n)} \quad \text { for } n \geq N,
$$

and for all constants $K>0$. 
Proof From $\left(H_{2}\right)$ and the definition of $z(n)$, we have $z(n) \geq x(n)$ for all $n \geq n_{1} \geq n_{0}$. Further, from Case (2) of Lemma 2.3, we have

$$
\Delta z(s) \leq \frac{a(n) \Delta z(n)}{a(s)}, \quad s \geq n .
$$

Summing the last inequality from $n$ to $j$, we obtain

$$
z(j+1)-z(n) \leq a(n) \Delta z(n) \sum_{s=n}^{j} \frac{1}{a(s)}
$$

and then letting $j \rightarrow \infty$, we obtain

$$
0 \leq z(n)+A(n) a(n) \Delta z(n), \quad n \geq n_{1} .
$$

Hence $\Delta\left(\frac{z(n)}{A(n)}\right) \geq 0$ for all $n \geq n_{1}$, and therefore $\frac{z(n)}{A(n)}$ is increasing for all $n \geq n_{1}$. From the definition of $z(n)$, we have

$$
\begin{aligned}
x(n) & =z(n)-\sum_{i=1}^{m} p_{i}(n) x^{\alpha_{i}}\left(n-k_{i}\right) \geq z(n)-\sum_{i=1}^{m} p_{i}(n) z^{\alpha_{i}}\left(n-k_{i}\right) \\
& \geq\left(1-\sum_{i=1}^{m} p_{i}(n) \frac{A^{\alpha_{i}}\left(n-k_{i}\right)}{A(n)} K^{\alpha_{i}-1}\right) z(n),
\end{aligned}
$$

where we have used $\frac{z(n)}{A(n)} \geq K$ and $\alpha_{i}>1$ for $i=1,2, \ldots, m$ and for all $n \geq N$.

We begin with the following oscillation result.

Theorem 2.1 Assume that $\left(H_{1}\right)-\left(H_{3}\right)$ and (1.4) hold. If $(1-P(n+1-\ell))>0$ for all $n \geq$ $N \geq n_{0}$ and

$$
\sum_{n=N}^{\infty} q(n)(1-P(n+1-\ell))^{\beta}=\infty, \quad n \geq N \geq n_{0}
$$

hold, then every solution of Eq. (1.1) is oscillatory.

Proof Let $\{x(n)\}$ be a positive solution of Eq. (1.1), say $x(n)>0, x\left(n-k_{i}\right)>0$ and $x(n+1-\ell)>$ 0 for $i=1,2, \ldots, m$ and for all $n \geq n_{1} \geq n_{0}$. From the definition $z(n)$, we have $z(n)>0$ for all $n \geq N \geq n_{1}$, where $N$ is chosen so that (2.1) holds for all $n \geq N$. From Lemma 2.2 and Eq. (1.1), we have

$$
\Delta(a(n) \Delta z(n))+q(n)(1-P(n+1-\ell))^{\beta} z^{\beta}(n+1-\ell) \leq 0, \quad n \geq N .
$$

Summing the last inequality from $N$ to $n$, we obtain

$$
\sum_{s=N}^{n} q(s)(1-P(s+1-\ell))^{\beta} \leq a(N) \Delta z(N) .
$$


Since $z(n) \geq M>0$, the last inequality contradicts (2.5) as $n \rightarrow \infty$. This completes the proof.

In the next theorem, we reduce the oscillation of Eq. (1.1) to that of a first order delay difference equation.

Theorem 2.2 Assume that $\left(H_{1}\right)-\left(H_{3}\right)$ and (1.4) hold. If $(1-P(n+1-\ell))>0$ for all $n \geq n_{0}$ and the delay difference equation

$$
\Delta w(n)+q(n)(1-P(n+1-\ell))^{\beta} R^{\beta}(n+1-\ell) w^{\beta}(n+1-\ell)=0, \quad n \geq n_{0}
$$

is oscillatory, then every solution of Eq. (1.1) is oscillatory.

Proof Let $\{x(n)\}$ be a positive solution of Eq. (1.1), say $x(n)>0, x\left(n-k_{i}\right)>0$ and $x(n+1-\ell)>$ 0 for $i=1,2, \ldots, m$ and for all $n \geq n_{1} \geq n_{0}$. Proceeding as in the proof of Theorem 2.1, we obtain (2.6). Now, using $z(n) \geq R(n) a(n) \Delta z(n)$, we have

$$
\Delta(a(n) \Delta z(n))+q(n)(1-P(n+1-\ell))^{\beta} R^{\beta}(n+1-\ell)(a(n+1-\ell) \Delta z(n+1-\ell))^{\beta} \leq 0
$$

for $n \geq N \geq n_{1}$. Set $w(n)=a(n) \Delta z(n)$. Then $\{w(n)\}$ is a positive solution of the inequality

$$
\Delta w(n)+q(n)(1-P(n+1-\ell))^{\beta} R^{\beta}(n+1-\ell) w^{\beta}(n+1-\ell) \leq 0, \quad n \geq N .
$$

It follows from Lemma 2.7 of [16] that the corresponding Eq. (2.7) also has a positive solution, which is a contradiction. The proof is now complete.

Remark 2.1 Employing sufficient conditions for the oscillation of all solutions of Eq. (2.7) for different values of $\beta$, one can obtain easily verifiable criteria for the oscillation of all solutions of Eq. (1.1).

Corollary 2.1 Assume $\left(H_{1}\right)-\left(H_{3}\right)$ and (1.4) hold. If $\beta=1, \ell \geq 2$ and

$$
\lim _{n \rightarrow \infty} \inf \sum_{s=n-\ell+1}^{n-1} q(s)(1-P(s+1-\ell)) R(s+1-\ell)>\left(\frac{\ell-1}{\ell}\right)^{\ell}
$$

hold, then every solution of Eq. (1.1) is oscillatory.

Proof Condition (2.8) and Theorem 7.5.1 of [3] imply oscillation of Eq. (2.7). The assertion now follows from Theorem 2.2. This completes the proof.

Corollary 2.2 Assume $\left(H_{1}\right)-\left(H_{3}\right)$ and (1.4) hold. If $\beta<1, \ell \geq 2$ and

$$
\sum_{n=N}^{\infty} q(n)(1-P(n+1-\ell))^{\beta} R^{\beta}(n+1-\ell)=\infty
$$

hold, then every solution of Eq. (1.1) is oscillatory. 
Proof Condition (2.9) and Theorem 1 of [11] imply oscillation of Eq. (2.7). The assertion now follows from Theorem 2.2, and this completes the proof.

Corollary 2.3 Assume that $\left(H_{1}\right)-\left(H_{3}\right)$ and (1.4) hold. If $\beta>1, \ell \geq 2$, and there exists $\lambda>$ $\frac{1}{\ell-1} \ln \beta$ such that

$$
\lim _{n \rightarrow \infty} \inf \left[q(n)(1-P(n+1-\ell))^{\beta} R^{\beta}(n+1-\ell) \exp \left(-e^{\lambda n}\right)\right]>0
$$

hold, then every solution of Eq. (1.1) is oscillatory.

Proof Condition (2.10) and Theorem 2 of [11] imply oscillation of Eq. (2.7). The assertion now follows from Theorem 2.2. This completes the proof.

Our next results are for the case where (1.5) holds in place of (1.4). We will also need the condition

$$
B(n)=(1-Q(n))>0
$$

for all $n \geq N \geq n_{0}$.

Theorem 2.3 Let $\beta>1$, and $\left(H_{1}\right)-\left(H_{3}\right),(1.5)$ and (2.11) hold. If there exists a positive nondecreasing real function $\{\rho(n)\}$ such that

$$
\lim _{n \rightarrow \infty} \sup \sum_{s=N}^{n}\left[\rho(s) q(s)(1-P(s+1-\ell))^{\beta} A^{\beta-1}(s+1-\ell)-\frac{a(s-\ell)(\Delta \rho(s))^{2}}{4 \rho(s)}\right]=\infty
$$

and

$$
\sum_{n=N}^{\infty} \frac{1}{a(n)} \sum_{s=N}^{n-1} q(s)(1-Q(s+1-\ell))^{\beta} A^{\beta}(s+1-\ell)=\infty
$$

hold, then every solution of Eq. (1.1) is oscillatory.

Proof Let $\{x(n)\}$ be a positive solution of Eq. (1.1) such that $x(n)>0, x\left(n-k_{i}\right)>0$ and $x(n+1-\ell)>0$ for $i=1,2, \ldots, m$ and for all $n \geq n_{1} \geq n_{0}$. From the definition of $z(n)$, we have $z(n)>0$ for all $n \geq N \geq n_{1}$, where $N$ is chosen so that two cases of Lemma 2.3 hold for all $n \geq N$. We shall show that in each case we are led to a contradiction.

Case (1) From the proof of Theorem 2.1, we see that (2.6) holds. Since $z(n)$ is increasing and $A(n)$ is decreasing and tending zero, we have $z(n) \geq A(n)$ for all $n \geq N$. Using this in (2.6), we obtain

$$
\Delta(a(n) \Delta z(n))+q(n)(1-P(n+1-\ell))^{\beta} A^{\beta-1}(n+1-\ell) z(n+1-\ell) \leq 0
$$

for all $n \geq N$ since $\beta>1$. Define

$$
w(n)=\rho(n) \frac{a(n) \Delta z(n)}{z(n-\ell)}, \quad n \geq N
$$


Then $w(n)>0$ for all $n \geq N$, and using (2.14), we obtain

$$
\begin{aligned}
\Delta w(n) \leq & -\rho(n)(1-P(n+1-\ell))^{\beta} A^{\beta-1}(n+1-\ell)+\frac{\Delta \rho(n)}{\rho(n+1)} w(n+1) \\
& -\frac{\rho(n)}{\rho(n+1)} \frac{w^{2}(n+1)}{a(n-\ell)}, \quad n \geq N .
\end{aligned}
$$

Applying the completing the square and then summing the resulting inequality, we obtain

$$
\sum_{s=N}^{n}\left[\rho(s)(1-P(s+1-\ell))^{\beta} A^{\beta-1}(s+1-\ell)-\frac{a(s-\ell)(\Delta \rho(s))^{2}}{4 \rho(s)}\right] \leq w(N) .
$$

Letting $n \rightarrow \infty$ in the above inequality, we get a contradiction with (2.12).

Case (2) From Eqs. (1.1) and (2.4), we have

$$
\Delta(a(n) \Delta z(n))+q(n)(1-Q(n+1-\ell))^{\beta} z^{\beta}(n+1-\ell) \leq 0, \quad n \geq N
$$

Summing the last inequality from $N$ to $n-1$, we obtain

$$
\frac{1}{a(n)} \sum_{s=n}^{n-1} q(s)(1-Q(s+1-\ell))^{\beta} z^{\beta}(s+1-\ell) \leq-\Delta z(n) .
$$

Summing again the last inequality, we get

$$
\sum_{s=N}^{n-1} \frac{1}{a(s)} \sum_{t=N}^{s-1} q(t)(1-Q(t+1-\ell))^{\beta} z^{\beta}(t+1-\ell) \leq z(N) .
$$

Since $\frac{z(n)}{A(n)}$ is increasing, there exists a constant $M>0$ such that $\frac{z(n)}{A(n)} \geq M$ for all $n \geq N$. Using this in (2.15) and letting $n \rightarrow \infty$, we obtain a contradiction with (2.13). This completes the proof.

Theorem 2.4 Let $\beta=1$, and $\left(H_{1}\right)-\left(H_{3}\right),(1.5)$ and (2.11) hold. If there exists a positive nondecreasing function $\{\rho(n)\}$ such that

$$
\lim _{n \rightarrow \infty} \sup \sum_{s=N}^{n}\left[\rho(s) q(s)(1-P(s+1-\ell))-\frac{a(s-\ell)(\Delta \rho(s))^{2}}{4 \rho(s)}\right]=\infty
$$

and

$$
\sum_{n=N}^{\infty} \frac{1}{a(n)} \sum_{s=N}^{n-1} q(s)(1-Q(s+1-\ell)) A(s+1-\ell)=\infty
$$

hold, then every solution of Eq. (1.1) is oscillatory.

Proof The proof is similar to that of Theorem 2.3, and hence it is omitted.

Theorem 2.5 Let $0<\beta<1$, and $\left(H_{1}\right)-\left(H_{3}\right)$, (1.5) and (2.11) hold. If conditions (2.9) and (2.13) hold, then every solution of Eq. (1.1) is oscillatory. 
Proof The proof follows from Corollary 2.2 and Theorem 2.3, and hence the details are omitted.

\section{Examples}

In this section, we present some examples to illustrate the main results.

Example 3.1 Consider the second order neutral difference equation

$$
\Delta\left(\frac{1}{n+1} \Delta\left(x(n)+\frac{1}{n^{5}} x^{3}(n-2)+\frac{1}{n^{9}} x^{5}(n-1)\right)\right)+n^{2} x^{5 / 3}(n-1)=0, \quad n \geq 1
$$

Here $a(n)=\frac{1}{n+1}, p_{1}(n)=\frac{1}{n^{5}}, p_{2}(n)=\frac{1}{n^{9}}, q(n)=n^{2}, \alpha_{1}=3, \alpha_{2}=5, \beta=\frac{5}{3}, k_{1}=2, k_{2}=1$, and $\ell=2$. Now $R(n)=\left(\frac{n^{2}+n-2}{2}\right), P(n)=\frac{M^{2}}{n^{5}}\left(\frac{n^{2}+n-2}{2}\right)^{2}+\frac{M^{4}}{n^{9}}\left(\frac{n^{2}+n-2}{2}\right)^{4}$, and $(1-P(n)) \geq 1-\frac{M^{2}}{4 n}-\frac{M^{4}}{16 n}>$ 0 for all $n \geq N$ where $N$ is sufficiently large. Further condition (2.5) becomes

$$
\sum_{n=N}^{\infty} q(n)(1-P(n+1-\ell))^{\beta} \geq \sum_{n=N}^{\infty} n^{2}\left(1-\frac{M^{2}}{4(n-1)}-\frac{M^{4}}{16(n-1)}\right)^{\frac{5}{3}}=\infty
$$

Hence all the conditions of Theorem 2.1 are satisfied, and therefore every solution of Eq. (3.1) is oscillatory.

Example 3.2 Consider the second order neutral difference equation

$$
\Delta^{2}\left(x(n)+\frac{1}{2^{n}} x^{7 / 3}(n-1)+\frac{1}{3^{n}} x^{3}(n-2)\right)+3^{n} x^{1 / 3}(n-1)=0, \quad n \geq 1 .
$$

Here $a(n)=1, p_{1}(n)=\frac{1}{2^{n}}, p_{2}(n)=\frac{1}{3^{n}}, q(n)=3^{n}, \alpha_{1}=\frac{7}{3}, \alpha_{2}=3, \beta=\frac{1}{3}, k_{1}=1, k_{2}=2$, and $\ell=2$. Now $R(n)=n, P(n)=\frac{M^{\frac{4}{3}}}{2^{n}} n^{\frac{4}{3}}+\frac{M^{2}}{3^{n}} n^{2}$, and $(1-P(n)) \geq\left(1-\frac{\left(M^{\frac{4}{3}}+M^{2}\right)}{3^{n}} n^{2}\right)>0$ for $n \geq N$ where $N$ is sufficiently large. Further condition (2.9) becomes

$$
\sum_{n=N}^{\infty} q(n)(1-P(n+1-\ell))^{\beta} R^{\beta}(n+1-\ell) \geq \sum_{n=N}^{\infty} 3^{n}\left(1-\frac{\left(M^{\frac{4}{3}}+M^{2}\right)}{3^{n}} n^{2}\right)(n-1)^{\frac{1}{3}}=\infty
$$

Hence all the conditions of Corollary 2.2 are satisfied, and therefore every solution of Eq. (3.2) is oscillatory.

Example 3.3 Consider the second order neutral difference equation

$$
\Delta\left(n(n+1) \Delta\left(x(n)+\frac{1}{n^{3}} x^{5 / 3}(n-1)+\frac{1}{n^{4}} x^{7 / 3}(n-2)\right)\right)+n^{3} x^{3}(n-1)=0, \quad n \geq 1 .
$$

Here $a(n)=n(n+1), p_{1}(n)=\frac{1}{n^{3}}, p_{2}(n)=\frac{1}{n^{4}}, q(n)=n^{3}, \alpha_{1}=\frac{5}{3}, \alpha_{2}=\frac{7}{3}, \beta=3, k_{1}=1, k_{2}=2$, and $\ell=2$. Now $R(n)=\frac{n-1}{n}$ and $A(n)=\frac{1}{n}$. Then

$$
P(n)=\frac{M^{\frac{2}{3}}}{n^{3}}\left(\frac{n-1}{n}\right)^{\frac{2}{3}}+\frac{M^{\frac{4}{3}}}{n^{4}}\left(\frac{n-1}{n}\right)^{\frac{4}{3}}<1 \quad \text { for } n \geq N
$$


and

$$
Q(n)=\frac{K^{2 / 3}}{n^{2}(n-1)^{2 / 3}}+\frac{K^{4 / 3}}{n^{3}(n-2)^{4 / 3}}<1 \quad \text { for } n \geq N,
$$

where $N$ is sufficiently large. By choosing $\rho(n)=1$, we see that condition (2.12) becomes

$$
\begin{aligned}
& \lim _{n \rightarrow \infty} \sup \sum_{s=N}^{n}\left[\rho(s) q(s)(1-P(s+1-\ell))^{\beta} A^{\beta-1}(s+1-\ell)\right] \\
& \geq \lim _{n \rightarrow \infty} \sup \sum_{s=N}^{n}\left(1-\frac{M^{\frac{2}{3}}+M^{\frac{4}{3}}}{s^{3}}\right)=\infty .
\end{aligned}
$$

Also condition (2.13) becomes

$$
\begin{aligned}
\sum_{n=N}^{\infty} \frac{1}{a(n)} \sum_{s=N}^{n-1} q(s)(1-Q(s+1-\ell))^{\beta} A^{\beta}(s+1-\ell) & \geq \sum_{n=N}^{\infty} \frac{1}{n(n+1)} \sum_{s=N}^{n-1}\left(1-\frac{K^{\frac{2}{3}}+K^{\frac{4}{3}}}{s^{2}}\right) \\
& \approx \sum_{n=N}^{\infty} \frac{1}{n+1}=\infty
\end{aligned}
$$

Hence all the conditions of Theorem 2.3 are satisfied, and therefore every solution of Eq. (3.3) is oscillatory.

\section{Conclusions}

In this paper, by using the Riccati transformation, the summation averaging technique and a new comparison theorem, we prove some suffcient conditions which are new, extend and complement some of the results established in $[2,5,6,9,10,12-18]$ either for the case $m=1$ or for the case $\alpha_{i}=1$ for $i=1,2,3, \ldots, m$. In particular, the results presented in $[9,15]$ cannot be applied to Eqs. (3.1) to (3.3) since $\alpha_{1} \neq 1$ and $\alpha_{2} \neq 1$. Further condition (2.11) is somewhat restrictive, and it implies that we must have $\left\{p_{i}(n)\right\} \rightarrow 0$ as $n \rightarrow \infty$ for $i=1,2, \ldots, m$. It would be good to obtain a result that did not require this added condition.

\section{Acknowledgements}

The authors are grateful to the anonymous referee for his suggestions, which have greatly improved the presentation of the paper.

\section{Funding}

This research work is not supported by any funding agencies.

\section{Competing interests}

The authors declare that they have no competing interests.

\section{Authors' contributions}

Both the authors have made equal contribution. Both the authors read and approved the final manuscript.

\section{Author details}

${ }^{1}$ Department of Mathematics, Government Arts College (Autonomous), Salem, India. ${ }^{2}$ Research and Development Center, Bharathiar University, Coimbatore, India.

\section{Publisher's Note}

Springer Nature remains neutral with regard to jurisdictional claims in published maps and institutional affiliations. 


\section{References}

1. Agarwal, R.P., Bohner, M., Grace, S.R., O'Regan, D.: Discrete Oscillation Theory. Hindawi Pub. Corp., New York (2005)

2. Dharuman, C., Graef, J.R., Thandapani, E., Vidhyaa, K.S.: Oscillation of second order difference equation with a sub-linear neutral term. J. Math. Appl. 40, 59-67 (2017)

3. Gyori, I., Ladas, G.: Oscillation Theory of Delay Differential Equations with Applications. Clarendon, Oxford (1991)

4. Hale, J.K.: Theory of Functional Differential Equations. Springer, New York (1977)

5. Kamaraj, B., Vasuki, R.: Oscillation of second order difference equation with a superlinear neutral term. J. Adv. Math. Comput. Sci. 23, 1-10 (2017)

6. Kamaraj, B., Vasuki, R.: Some oscillation results for second order difference equation with a sublinear neutral term. Pac.-Asian J. Math. 4, 100-109 (2017)

7. Liu, X.: Oscillation of solutions of neutral difference equations with nonlinear term. Comput. Math. Appl. 52, 439-448 (2006)

8. MacDonald, N.: Biological Delay Systems: Linear Stability Theory. Cambridge University Press, Cambridge (1989)

9. Selvarangam, S., Rupadevi, S.A., Thandapani, E.: Oscillation theorems for second order quasilinear difference equations with linear neutral terms. Transylv. J. Math. Mech. 9, 63-72 (2017)

10. Sun, Y.G., Saker, S.H.: Oscillation of second order nonlinear neutral delay differene equaitons. Appl. Math. Comput. $163,909-918(2005)$

11. Tang, X.H., Liu, Y.: Oscillation for nonlinear delay difference equations. Tamkang J. Math. 32, 275-280 (2001)

12. Thandapani, E., Mahalingam, K.: Necessary and sufficient conditions for oscillation of second order neutral difference equation. Tamkang J. Math. 34, 137-145 (2003)

13. Thandapani, E., Mahalingam, K., Graef, J.R.: Oscillatory and asymptotic behavior of second order neutral type difference equations. Int. J. Pure Appl. Math. 6, 217-230 (2003)

14. Thandapani, E., Pandian, S., Balasubramanian, R.K.: Oscillation of solutions of nonlinear neutral difference equations with nonlinear neutral term. Far East J. Appl. Math. 15, 47-62 (2004)

15. Thandapani, E., Seghar, D., Selvarangam, S.: Oscillation of second order quasilinear difference equations with several neutral terms. Transylv. J. Math. Mech. 7, 67-74 (2015)

16. Thandapani, E., Selvarangam, S.: Oscillation theorems of second order quasilinear neutral difference equations. J. Math. Comput. Sci. 2, 866-879 (2012)

17. Yildiz, M.K., Ogunmez, H.: Oscillation results of higher order nonlinear neutral delay difference equations with a nonlinear neutral term. Hacet. J. Math. Stat. 43, 809-814 (2004)

18. Zhang, Z., Chen, J., Zhang, C.: Oscillation of solutions for second order nonlinear difference equations with nonlinear neutral term. Comput. Math. Appl. 41, 1487-1494 (2001)

\section{Submit your manuscript to a SpringerOpen ${ }^{\circ}$ journal and benefit from:}

- Convenient online submission

- Rigorous peer review

- Open access: articles freely available online

- High visibility within the field

- Retaining the copyright to your article

Submit your next manuscript at $>$ springeropen.com 